\title{
Corrigendum to: Thinking twice about sum scores
}

\section{Daniel McNeish ${ }^{1} \cdot$ Melissa Gordon Wolf ${ }^{2}$}

Published online: 31 August 2020

(C) The Psychonomic Society, Inc. 2020

Since publication, it has come to our attention that there are a few errors present in the article. The supplemental materials posted on the Open Science Framework (https://osf.io/cahtb/) were correct but were not necessarily described correctly in the final version of the article. All errors were made by the first author. We use pdf page numbers because the article has not yet been assigned to an issue and does not have official page numbers at this time.

1. In Table 2 on page 6 of the pdf, the decimal point for the error variance of Item 6 was misplaced. The error variance for Item 6 is listed as 0.11 , but the correct value is 1.08 .

2. The chi-square statistic on page 8 of the pdf is listed as 5361.86; the correct chi-square value is 325.90 . The conclusion does not change, because $p<.01$ in either case.

3 . In the 'How scoring approaches can change conclusions' section that begins on page 13 of the pdf, we flipped the labels for the Grant-White and Pasteur schools such that the effects would be interpreted to be in the opposite direction. The descriptions of the school differences should be reversed. The data in this section were an example that did not produce substantive conclusions, and arguments in the paper do not materially change after reversing the school labels.

4. The $p$-value on page 14 of the pdf for school differences on the sum score was reported to be equal to .05 when rounded to two decimal points. If rounding to three decimal points, the $p$-value would be .054 . The discordance between a sum score of all six items (Method 1) and using either factor score regression (Method 3) or the simultaneous approach (Method 4) remains whether the school effect with sum scores is significant or not, because there are school differences on Verbal Cognition but not on Speeded Cognition in Method 3 and Method 4, either way demonstrating how different scoring choices can lead to different conclusions.

We appreciate the attention of William Revelle, John Sakaluk, and Keith Widaman for noting these errors in the text.

Publisher's note Springer Nature remains neutral with regard to jurisdictional claims in published maps and institutional affiliations.
Daniel McNeish

dmcneish@asu.edu

1 Arizona State University, Phoenix, AZ, USA

2 University of California, Santa Barbara, Santa Barbara, CA, USA 